Rev. salud pública. 14 sup (1): 56-67, 2012

\title{
Los problemas en el mundo del trabajo y su impacto en salud. Crisis financiera actual
}

\author{
Problems of work world and its impact on health \\ Current financial crisis
}

\author{
Fernando Tomasina \\ Facultad de Medicina. Universidad de la República. Uruguay. fernandotomasina@gmail.com \\ Recibido 26 Septiembre 2009/Enviado para Modificación 3 Marzo 2010/ Aceptado 10 Mayo 2010
}

\section{RESUMEN}

La salud y el trabajo son procesos múltiples y complejos, vinculados e influenciados entre sí. Se puede afirmar que el mundo del trabajo es extremadamente complejo y heterogéneo en donde conviven los llamados "viejos" o tradicionales riesgos con los derivados de los nuevos modelos de organización laboral y de la incorporación de las nuevas tecnologías, los llamados "riesgos modernos". Las estrategias neoliberales han tenido como resultados la desocupación, la precarización de las relaciones laborales y la externalización de los riesgos laborales. En el actual trabajo se presentan algunos de los emergentes negativos del proceso de salud-enfermedad observados a partir de estas transformaciones en el mundo del trabajo y además de los derivados de la actual situación de crisis económica mundial. Por último se plantea la necesidad de reconstrucción de políticas que atiendan esta problemática derivada del mundo del trabajo.

Palabras Clave: Salud, trabajo, crisis, desempleo, riesgos laborales (fuente: DeCS, BIREME)

\section{ABSTRACT}

Health and work are complex processes. Besides, they are multiple considering the forms they take. These two processes are linked to each other and they are influenced by each other. According to this, it is possible to establish that work world is extremely complex and heterogenous. In this world, "old" or traditional risks coexist with "modern risks", derived from the new models of work organization and the incorporation of new technologies. Unemployment, work relationships precariousness and work risks outsourcing are results of neoliberal strategies. Some negative results of healthsickness process derived from transformation in work world and current global economic crisis have been noticed in current work conditions. Finally, the need for reconstructing policies focusing on this situation derived from work world is suggested. 
Key Words: Health, work, crisis, unemployment, occupational accidents (source: Mesh, NLM) a salud y el trabajo son procesos múltiples y complejos, vinculados e influenciados entre sí. El trabajo aún en condiciones adversas es un mecanismo que permite el desarrollo de varias destrezas del ser humano, por lo que se puede afirmar siempre la existencia de un polo positivo del trabajo, generador de bienestar, en definitiva de salud.

Si bien más comúnmente se toman en cuenta solamente los aspectos negativos del trabajo sobre la salud de los trabajadores, por ejemplo los accidentes de trabajo, la gravedad de los mismos, las enfermedades ocupacionales, etc., no se debe olvidar los aspectos positivos que conlleva el proceso de trabajo (identificación social, sentido de pertenencia, etc.) (1).

El proceso salud-enfermedad está determinado, en gran medida, por los espacios de consumo y producción en los cuales los colectivos de trabajadores están insertos socialmente. Esto explica los diferentes perfiles epidemiológicos de salud-enfermedad en los diferentes grupos de clases sociales, ello se corresponde con la característica del grupo social al que pertenece y con las particularidades de cada individuo.

Al estudiar la salud-enfermedad de la población trabajadora, es adecuado retomar lo planteado por Breilh, al establecer diferentes dominios de análisis. Un dominio general (estructurales: producción y consumo, político y cultura), otro particular (mediadores grupales: género, reproducción familiar, organización gremial) y uno singular (genotipofenotipo). Para cada uno de los diferentes dominios se necesitan diferentes estrategias orientadas a la promoción de la salud, con una lógica común: Promover los procesos saludables existentes y así mismo controlar y o eliminar los procesos destructivos (2).

Diferentes formas de organización laboral se han presentado en distintos momentos históricos. En el Uruguay, como prácticamente en todos los países de la región, convergen desde las formas más simples hasta las más complejas. Se puede afirmar que el mundo del trabajo es extremadamente complejo y heterogéneo en donde conviven los llamados 
"viejos" o tradicionales riesgos con los derivados de los nuevos modelos de organización laboral y de la incorporación de las nuevas tecnologías, los llamados "riesgos modernos".

Solo hay que detenerse a pensar en lo que significan las Tecnologías de la Información y Comunicación (TIC), cómo han generado profundos cambios en el proceso productivo al interactuar con el colectivo de trabajadores, afectando diferentes dimensiones: lo familiar, lo laboral, lo sindical, inclusive el entorno general de vida. Quizás lo más notable sea la "contaminación” del espacio laboral en el espacio cotidiano de la vida como se presenta en el tele-trabajo al hacer uso de este tipo de tecnología en su forma más radicalizada, el tele-trabajo realizado en forma independiente desde el hogar (3). De esta manera, las TIC como tecnología se incorporaron frecuentemente a las ya existentes malas condiciones de trabajo, por ejemplo desde la perspectiva ergonómica, con baja remuneración y contratos precarios.

\section{TRABAJO PRECARIO, DESOCUPACIÓN Y SALUD}

Tres conceptos, trabajo precario, desocupación y salud, que es necesario vincularlos adecuadamente; los tres como fenómenos sociales se desarrollan en una misma lógica, la del actual modelo económico-productivo mundializado. No son pues fenómenos aislados, ni el desempleo, ni la precarización de las condiciones de trabajo, ni el producto de ellos: la salud.

Desempleo y precarización son fenómenos esperados en los procesos de producción dependientes de otro proceso: el de valorización, es decir de generación de ganancias (4); claramente amplificado dicho fenómeno por las políticas económicas neoliberales impuestas a los países dependientes y subdesarrollados, en el marco además de la globalización. La actual situación de crisis financiera mundial ha disparado nuevamente los temores de agudización de los problemas en el trabajo y la salud de la población. La Directora General de la Organización Mundial de la Salud, Margaret Chan, ha declarado que: "Los países de todos los niveles de desarrollo económico están preocupados por el impacto de la crisis financiera en la salud. Si continúa aumentando el desempleo, si fallan las redes de protección social, si se erosionan los ahorros y los fondos de pensiones y si caen los gastos públicos, es inevitable que la salud de la gente sufra”. Y añade: "El impacto es directo cuando el estrés causa un aumento del las enfermedades 
mentales y el uso del tabaco, el alcohol y otras sustancias dañinas. Y todo empeora cuando los servicios sanitarios no pueden mantener los cuidados que necesitan los enfermos” (5).

Precarización y desocupación han sido los síntomas de esta enfermedad, ambos hechos sociales están íntimamente relacionados y actúan prácticamente como un par dialéctico. Como afirmó Rodríguez: “Nadie ignora que estas patologías del trabajo, permítanme llamarlas así pues creo que el trabajo en negro, la temporalidad o la eventualidad merecen encuadrarse como tales, crecieron desmesuradamente, no crecieron solas, sino a la luz de un derecho laboral de apoyo irrestricto a las doctrinas neoliberales y bajo la bandera de la llamada flexibilidad laboral” (6).

Se puede afirmar que las estrategias neoliberales, sin duda orientadas para lograr mayor extracción del valor, han tenido como resultados en el mundo del trabajo la desocupación, la precarización de las relaciones laborales, la externalización de los riesgos laborales, la tercerización y el debilitamiento de las funciones del Estado en cuanto a regulador de las siempre desiguales relaciones entre el trabajador y el empleador. A manera de ejemplo pude mencionarse la exposición a plaguicidas sin ningún criterio de seguridad, a los que han sido expuestos frecuentemente los trabajadores rurales en su condición de temporales. La exposición masiva a solventes en varios pequeños talleres para la producción y elaboración de partes de prendas de cueros que responden a grandes empresas, pero sin responsabilidad sobre los trabajadores. Este último ejemplo responde a la extensión de modelos de producción segmentada con externalización de parte del proceso productivo realizado en pequeños talleres cuasi familiares, sin los controles ambientales correspondientes.

En lo referente a la externalización tal como afirma Rodríguez, es también una externalización del riesgo (6). Procesos productivos penosos muy contaminantes se dan a terceros como forma de disminuir costos y/o responsabilidades vinculadas a las acciones en salud y seguridad en el trabajo. Por otra parte, la desocupación creciente actúa como forma de chantaje social sobre los ocupados, para estos resulta ser su posible futuro, hay por tanto que aceptar la rebaja salarial, la flexibilización de las condiciones laborales puesto que el único camino es la desocupación. Al decir de Aguiar la desocupación es una amenaza colectiva, estructural y desocializada (7). Esta última afirmación se sustenta en que el desempleo, que si bien es un fenómeno social, es vivido como crisis individual despojado de su dimensión social. 
De alguna manera existe la institucionalización del desempleo, con la consiguiente aceptación, conformismo y resignación de condiciones de trabajo poco dignas. La OMS en 1986 ubicaba al desempleo como la principal catástrofe epidemiológica de nuestro tiempo (7). Hoy dicha situación está agudizada dado el crecimiento de este fenómeno, así como el aumento de la precariedad laboral. Este último oficia de desempleo encubierto como afirma el economista Daniel Olesker, ya que no satisface las necesidades, económicas, sociales y laborales de las personas (8).

La precarización de las condiciones de trabajo se extiende hasta la vida misma de las personas y resulta ser uno de los síntomas de la enfermedad social que, al igual que las enfermedades trasmisibles, ha sido trasmitida como pandemia y ha ido afectando a los más susceptibles, es decir a los más débiles socialmente.

No solo el espacio social de producción del trabajador se afecta sino que también el espacio social de consumo. Como muestra del mismo se accede a menor cantidad de servicios y de menor calidad. La enseñanza, la alimentación, la vivienda, entre otros, son también servicios y o bienes precarizados para el trabajador precarizado.

Síndromes del modelo productivo impuesto

Se han utilizado diferentes síndromes para caracterizar expresiones de salud- enfermedad emergentes entre los trabajadores pero en común tienen una misma lógica de producción. Se presentan estos "emergentes" probablemente vinculados a los nuevos modelos de organización y de producción laboral que conviven con viejos modelos laborales, pero que en estos últimos, como suerte de "modernización" se incorporan formas "nuevas” (flexibilización, desregulación, externalización).

Existen diferentes investigaciones que ponen de manifiesto efectos en la salud de los trabajadores en relación con algunas dimensiones de la precariedad como es el empleo temporal vinculado con la siniestralidad y la afección en el área de la salud mental $(9,10)$. De acuerdo con la investigación conducida por Amable, Benach y González sobre la precariedad laboral y su repercusión sobre la salud, "los problemas de salud mental que aparecen más estrechamente asociados a la precariedad laboral son el nerviosismo y la ansiedad, la depresión, el miedo, el sufrimiento, la sociabilidad y las relaciones de amistad” (9). 
A continuación se presentan algunos de los emergentes negativos del proceso de salud-enfermedad, observados a partir de estas transformaciones en el mundo del trabajo y que además es probable que se profundicen en la actual situación de crisis económica mundial:

Síndrome del Parado: Expresión utilizada frecuentemente por autores europeos, que identifica un conjunto de signos y síntomas sicofísicos que ocurren en el trabajador que se encuentra desempleado. Se describen tres fases, una primera fase de signo lúdico, en que el parado se encuentra optimista con tiempo para realizar actividades que no podía por el trabajo. Una segunda fase caracterizada por la búsqueda de empleo; la ansiedad y angustia dominan esta etapa. Las alteraciones digestivas y cardiovasculares son frecuentes. Por último existe una fase de depresión. El trabajador en esta fase considera que el problema es de él mismo, se siente desvalorizado, en crisis de autoestima. Es marginado y se automargina. El desempleo significa para el trabajador pérdidas múltiples en diferentes dimensiones de su ser (11).

De acuerdo a una investigación realizada en el Uruguay, por el equipo de investigadores que lidera Araujo, los principales efectos psicosociales de la desocupación en mayores de 40 años son la depresión, pérdida de autoestima y de sentido de pertenencia (12).

Accidentes laborales: No resulta ser una nueva patología ocupacional, pero se ha observado un aumento de los mismos en aquellos sectores donde se ha profundizado el modelo productivo con precarización de las condiciones laborales. La precariedad laboral se ha asociado con mayores tasas de accidentes laborales. Esta siniestrabilidad aumentada está determinada por el modelo de trabajo basado en la temporalidad, movilidad permanente, ocupación irregular que conlleva una población de trabajadores fluctuante expuesta a permanentes alteraciones de riesgos laborales (13). A manera de ejemplo, en el trabajo de investigación realizado en Argentina, dirigido por Kohen de la Universidad Nacional de Rosario, observó que con el aumento de la desocupación y la profundización de la política de flexibilización y precarización laboral ha existido un aumento en los accidentes laborales en un $18 \%$ y de los mortales en un $11 \%$, comparando el último cuatrimestre del año 2000 con igual período del año anterior. 
Síndrome de Burnout: La precarización laboral y la consiguiente búsqueda de la utópica excelencia para no salir del mercado laboral, compitiendo permanentemente y siendo demando en forma continua ha determinado la aparición de este síndrome descrito en 1986 por Maslasch y Jackson. Observado principalmente en sectores de servicios como enseñanza y/o salud, se puede caracterizar el mismo por un síndrome de agotamiento emocional, despersonalización y bajos sentimientos de realización personal (14). En el trabajo de investigación sobre la salud de los docentes de educación primaria realizado en 2004 en 6 países de América Latina, se evidenció la presencia del síndrome dentro de los docentes (15). En el caso de Uruguay se observó, además, que los maestros de escuelas de contexto crítico y/o de tiempo completo presentan un riesgo aumentado. A partir de la aplicación del formulario adaptado MBI para medición de presencia del Síndrome de Burn Out (desgaste profesional) se observó la presencia en el 7 \% del personal docente, destacándose que las escuelas en entornos sociales desfavorables son un claro factor de riesgo para la aparición del desgaste profesional (Odds Ratio 4,11 Test exacto de Fisher 0,048).

Estrés: Sin duda la situación de desempleo, con búsqueda activa del trabajo, sin lograr resultados favorables es generadora de alta carga de estrés. Así mismo, las condiciones de inestabilidad laboral por inseguridad contractual son estresores sociales de relevancia. Si además se dan, como frecuentemente se observa, en malas condiciones de higiene y seguridad laboral, el estrés se intensifica. Clásicamente se define al estrés como una reacción de alerta frente a un estimulo externo. Dicha situación cuando se prolonga en el tiempo sobrecargando o excediendo las capacidades adaptativas del individuo, se manifiesta con diferente sintomatología, en general psicosomática. Las condiciones de trabajo precarias así como el desempleo operan como un factor estresante. Los problemas de salud mental que se han asociados con las condiciones de precariedad laboral son "la ansiedad, la depresión, el miedo, el sufrimiento, la sociabilidad y las relaciones de amistad” (10).

Lesiones a esfuerzos repetitivos (L.E.R.) y expresiones de morbilidad inespecíficas por exposición a múltiples contaminantes, son otras expresiones de la desregulación y polivalencia observadas en estos modelo de organización laboral. Los primeros (LER) por modelo de organización que no respetan los tiempos fisiológicos de trabajo y de descanso que 
frecuentemente están acompañados de un estímulo económico al trabajador, con pago por producción (destajo). Por otra parte la flexibilidad y la polivalencia determinan la poli exposición, que frecuentemente generan diferentes expresiones de malestar o enfermedad, ya no específicas, como clásicamente se describían en el ámbito de la medicina del trabajo.

Patología degenerativa de columna vertebral: Por uso extremo, continuo del aparato locomotor. Se observa, por ejemplo, en el trabajador rural y de la construcción, a los que se les asignan tareas que demandan gran esfuerzo físico con levantamiento de peso y posturas disergonómicas. Sin duda responde a la persistencia de formas tradicionales de trabajo, con baja inversión tecnológica.

La violencia en el lugar de trabajo: Para la OIT, la violencia en el trabajo es entendida como "Toda acción, incidente o comportamiento que se aparta de lo razonable mediante la cual una persona es agredida, amenazada, humillada o lesionada por otra en el ejercicio de su actividad profesional o como 'consecuencia directa' de la misma”. Está presente en diferentes ámbitos laborales. Como ejemplo del mismo, en el sector salud, en relación a los médicos residentes, se describieron situaciones de violencia en el trabajo en una encuesta realizada en el 2007. Dicha situación estaba presente en el $69 \%$ de los encuestados y habían vivido, por lo menos, una situación de violencia en el último año. Constituyéndose esta situación en una exigencia adicional a las tareas propias de su práctica médica (16). Diferentes formas de violencia en el trabajo son relatadas por los trabajadores de distintos sectores; una de las que ha tenido mayor presencia ha sido el denominado acoso moral o mobbing. Como afirma la Dra. Ana Sotelo existe acoso moral en el trabajo "toda vez que estamos ante hechos, actos u omisiones reiterados, que configuran maltrato, injuria, amenazas o cualquier tipo de conducta degradante del empleador o de cualquiera de sus dependientes, por los cuáles se configura la violación del derecho a la dignidad, el derecho a la integridad moral, a la intimidad, a la imagen, a un medio de trabajo adecuado, entre otros, produciendo un daño” (17).

Impacto en el mundo del trabajo en el Uruguay (18)

Uruguay tiene una población de 3314466 habitantes (1 601024 hombres y 1713442 mujeres). Abarca un área de 176215 km². Aproximadamente el 46 \% de la población se localiza en la capital del país, Montevideo, el 38 \% en el interior urbano, mientras que sólo 16 \% habita las zonas rurales. 
Durante las últimas décadas, en particular con el predominio de las políticas neoliberales, el país sufrió una importante desindustrialización. Durante ese periodo las políticas neoliberales en gran medida, y paradójicamente, facilitadas por el desempleo, determinaron condiciones de trabajo poco decentes y lejos de ser saludables. Un aspecto central que acompañó a esta política fue el retiro del Estado como ente regulador y/o de control de las condiciones laborales lo que ha sido parte del desafío que tuvo que asumir el actual gobierno.

La fuerte recesión económica, generó en los años 2001 a 2003 un descenso importante del ingreso promedio de los hogares. En el año 2004 este ingreso comenzó a aumentar, tendencia que se ha mantenido hasta el año 2008. Con respecto a la tasa de desempleo, la evolución observada fue de valores cercanos al $20 \%$, en plena crisis, a valores menores a un dígito actualmente.

Según el INE "la estimación de la Tasa de Desempleo para el total del país se ubicó en agosto 2008 en 7,6 \%. En Montevideo se estimó en 6,9 y en el Interior 8,3 \%, lo que marca con respecto a julio 2008 una disminución del desempleo de un punto porcentual en Montevideo y un aumento de igual nivel en el interior. Si se compara con igual período del año anterior, la tasa de desempleo disminuye en todas las áreas consideradas, siendo esta disminución más acentuada en Montevideo donde el desempleo baja 1,4 puntos porcentuales. Las tasas específicas de desempleo para hombres y mujeres se estimaron en esta oportunidad en 6,0 y 9,5 por ciento respectivamente, lo que marca con respecto a agosto de 2007 una disminución de la desocupación de 2 puntos porcentuales en las mujeres e igual nivel en los hombres"(19).

El Estado Uruguayo tuvo una fuerte impronta de protección al trabajador a principios del siglo veinte, en el marco del Estado de Bienestar, en relación a la legislación protectora de la salud del trabajador desde el año 1914, específicamente a través de la Ley de responsabilidad de empleadores 5 032/14 sobre accidentes y enfermedades del trabajo. En dicha ley se señala la responsabilidad de los empleadores en cuanto a la salud y seguridad de sus trabajadores, con un grado variable de cumplimiento de la misma.

En los años de neoliberalismo se impuso un esquema de desmantelamiento de los aparatos de control del Estado, liberando las relaciones laborales a las fuerzas del mercado; existió además una fuerte privatización de diferentes instituciones (entes) estatales, que fue parcialmente controlada por la presión social de diferentes actores. Cabe mencionar que en los últimos cuatro años, con el nuevo gobierno de orientación progresista, han 
existido diferentes acciones para revertir las consecuencias negativas de las políticas neoliberales en el mundo del trabajo, antes mencionadas.

En este sentido se realizó una acción directa para combatir el trabajo no registrado, comúnmente denominado en negro, aumentado la capacidad de control por parte de los organismos competentes en esa área (Instituto de seguridad Social, Ministerio de Trabajo Seguridad Social). Ejemplo de ello fue la instalación de la negociación colectiva en el trabajo (consejos de salarios), recreándose espacios de negociación paritaria y/o tripartita. Como otra muestra de esta voluntad, en el área de la salud y el trabajo se ha reglamentado el convenio internacional de OIT $\mathrm{N}^{\circ} 155$, habilitando la formación de las comisiones de salud y seguridad en el trabajo con integración en forma paritaria.

En cuanto al aspecto de la atención a la salud del trabajador, el país tiene un seguro de accidentes y enfermedad profesional, estatal y monopólica, ejercida por el Banco de Seguros de Estado (B.S.E), reglamentada por la Ley 16 074, referida al Seguro de Accidentes de Trabajo y las Enfermedades Profesionales. Esta ley no contempla la universalidad del seguro para todos los trabajadores, por tanto sigue siendo un tema pendiente de equidad en el sector. Por otra parte, el enfoque de atención a la salud de los trabajadores que ha tendido históricamente el país es predominantemente curativo e indemnizatorio.

El trabajo sigue siendo una actividad central para el ser humano, más allá de augurios de algunos pensadores contemporáneos que afirmaban la pérdida de la centralidad del trabajo y aún más su fin. El trabajo sigue estando íntimamente vinculado con el grado de bienestar psicofísico y social del individuo y del grupo al que pertenece (20).

La discusión es, en qué condiciones se realiza el mismo, si está en armonía con las expectativas del trabajador, si construye con él relaciones sociales beneficiosas, si le permite hacer uso de sus cualidades, si desarrolla sus potencialidades. La pregunta es entonces: ¿Es posible que el trabajo sea un generador de salud y no de sufrimiento, enfermedad o muerte, en las actuales condiciones? Seguramente hay varias respuestas, pero ninguna sería simple, lo que es claro es que se debe tratar de avanzar en el desarrollo de políticas en el trabajo que garanticen los derechos básicos del trabajador.

Como menciona Uriarte es necesario encarar "una reconstrucción de la 
protección del trabajador. Algunos elementos de ese modelo podrían ser la revalorización de la continuidad de la relación de trabajo, la capacitación, el desarrollo de los derechos laborales como derechos humanos, la acción internacional y la recuperación de la seguridad social” $(6,21)$. Estas reformas deberían estar orientadas por el derecho al trabajo, valorizando el mismo como propio de la condición humana, en la lógica de generar trabajo digno, decente. El trabajo, en estas condiciones, continúa siendo un formidable instrumento de promoción de la salud \&

\section{REFERENCIAS}

1. Tomasina F, Stolovas N. Condiciones de Trabajo y Salud II. Montevideo: Departamento de Salud Ocupacional. Facultad de Medicina. Universidad de la República; Diciembre 2009.

2. Breilh J. Nuevos conceptos y técnicas de investigación. Guía pedagógica para un taller de metodología. Quito: Centro de estudios y asesoría en salud (CEAS); 1995.

3. Blanco A. El Teletrabajo ¿oportunidad o amenaza? En: Sotelo A (Coord.) Curso Las Transformaciones en el Mundo del Trabajo y los desafíos del movimiento sindical. Montevideo: Red Académica de Trabajo. Comisión Sectorial de Educación Permanente. Udelar; 2008.

4. Laurell AC, Noriega M. La Salud en la Fábrica. México: Ediciones Era; 1989.

5. Rojas-Ochoa F [Internet]. Crisis y Desempleo. Disponible en: bvs.sld.cu/revistas/spu/ vol35_3_09/spu01309.htm. Consultado: Septiembre del 2009.

6. Rodríguez CA [Internet]. Cierre de la VI Semana Argentina de la Salud y Seguridad en el Trabajo Santa Fe, 28 de abril 2009. Disponible en: http://www.lla.org.pe/publicaciones/ docs/crisis/cierre_VI_Semana.pdf. Consultado: septiembre de 2009.

7. Aguiar E [Internet]. La desocupación: algunas reflexiones sobre sus repercusiones psicosociales. Disponible en: www.psiconet.com/foros/trabajo/desocupacion2.htm Consultado: septiembre de 2009.

8. Tomasina F. Trabajo precario, desocupación y salud. Psicología y organización del trabajo III. Montevideo: Editorial Psicolibros; 2002.

9. Amable M [Internet]. La precariedad laboral y su impacto en la salud. Un estudio en trabajadores asalariados en España. Disponible en: www.tesisenxarxa.net/TESIS_ UPF/AVAILABLE/TDX-0209109-142541//tma.pdf. Consultado: septiembre de 2009.

10. Amable M, Benach J, González S. La precariedad laboral y su repercusión sobre la salud: conceptos y resultados preliminares de un estudio multimétodos. Arch. Prev. Riesgos Labor. 2001; 4(4):169-184.

11. Carlotto MS, Gobbi MDr. Desempleo y Síndrome de Burnout. Revista de Psicología [Internet]. Disponible en: http://redalyc.uaemex.mx/redalyc/src/inicio/ArtPdfRed. jsp?iCve=26410110. Consultado: 20 de junio de 2009.

12. Araujo AM. Impactos imaginarios En: Araujo AM. (Coord.) Impactos del Desempleo: Transformaciones en la subjetividad. Montevideo: Argos; 2002

13. Ruiz-Frutos C. Salud laboral: conceptos y técnicas para la prevención de riesgos laborales. $3^{\mathrm{a}}$ Edn. Barcelona: Masson, Elsevier; 2007.

14. Ortega-Bayon JL. Incidencia del estrés del Síndrome de Burnout en los servicios de enfermería en cuidados intensivos. En: Valdivieso JD, Vallejo R (Coord.). Psicología del Trabajo. Madrid: Ediciones Pirámide S.A.; 1998.

15. UNESCO. Oficina Regional de Educación para América Latina y el Caribe-OREALC. Condiciones de Trabajo y Salud Docente. Estudios de casos en Argentina, Chile, Ecuador, México, Perú y Uruguay. Montevideo: Oficina del Libro. FEFMUR; 2006. 
16. Stolovas N, Tomasina F, Frantchez V, Pintos M, Aguirrezabal X. Una Visión de Género: Trabajadores Médicos Jóvenes en el Ejercicio de la Residencia. IV Semana Argentina de Salud y Seguridad en el Trabajo, SRT, Ministerio de Trabajo, Empleo y Seguridad Social.

Buenos Aires 2008 [Internet]. Disponible en: http://www.dso.fmed.edu.uy/archivos_saludocup/ Contenido/Material/MaterialInvestigacion/archivos/Residentes.pdf. Consultado: julio 2009.

17. Sotelo-Márquez A. Riesgos en la Organización del Trabajo. Condiciones Laborales y Organización del trabajo. Montevideo: Red Académica de Trabajo. Comisión Sectorial de Educación Permanente. Udelar; 2008.

18. Tomasina F, Stolovas N, Chaves, Arroyo A, Bozzo E, Miglionico W. Situación en Uruguay Informe Continental sobre la situación del Derecho a la Salud en el Trabajo, 2008. ALAMES [Internet] Disponible en: http://www.alames.org/documentos/urutra.pdf Consultado: Julio 2009.

19. Sociedad Uruguaya [Internet]. Disponible en: www.sociedaduruguaya.org/2008/10/ineactividad-empleo-y-desempleo-en-agosto-2008.html. Consultado: Junio 2009.

20. Bernheim R, Coiro M, Fernández R, García A. Empleo-Desempleo: ¿Mecanismo sin salida? En: Autores varios. Jornadas Universitarias Multidisciplinarias sobre las Transformaciones en el Mundo del Trabajo. La Práctica Multidisciplinaria en la Organización del Trabajo. Montevideo: Editorial Psicolibros; 2003.

21. Ermida-Uriarte O. La política laboral de los gobiernos progresistas. Revista Nueva Sociedad. 2007; 211: 50-65. 